\title{
\begin{tabular}{l|l} 
POLITIQUES \& & Politiques et management public
\end{tabular}
}

\section{Les obstacles à l'apprentissage organisationnel au sein de la sécurité civile : une mise en perspective en termes de gestion des ressources humaines}

How organizational learning dynamics hit obstacles : the case of the civil protection service and its HR management

Anaïs Gautier, Pascal Lièvre et Géraldine Rix-Lièvre

\section{OpenEdition}

\section{Journals}

Édition électronique

URL : http://journals.openedition.org/pmp/1438

DOI : $10.4000 / p m p .1438$

ISSN : 2119-4831

\section{Éditeur}

Institut de Management Public (IDPM)

\section{Édition imprimée}

Date de publication : 1 juin 2008

Pagination : 137-168

ISSN : 0758-1726

\section{Référence électronique}

Anaïs Gautier, Pascal Lièvre et Géraldine Rix-Lièvre, « Les obstacles à l'apprentissage organisationnel au sein de la sécurité civile : une mise en perspective en termes de gestion des ressources 


\title{
LES OBSTACLES À L'APPRENTISSAGE ORGANISATIONNEL AU SEIN DE LA SÉCURITÉ CIVILE : UNE MISE EN PERSPECTIVE EN TERMES DE GESTION DES RESSOURCES HUMAINES
}

\author{
Anaïs GAUTIER \\ Pascal LIĖVRE \\ Géraldine RIX-LIEVRE
}

\begin{abstract}
Résumé
L'objet de cette contribution est d'identifier un certain nombre de facteurs " humains " qui font obstacle à l'apprentissage organisationnel au sein de la sécurité civile. Notre intérêt se porte sur la première étape du processus d'apprentissage à savoir la perception d'une erreur par au moins un acteur en situation (Argyris, Schön, Senge). A partir de l'observation in situ d'une opération d'un Service Secours Incendie suite à un départ de feu dans le Sud de la France, nous avons pu identifier quatre registres qui peuvent constituer autant d'obstacles à l'apprentissage organisationnel. Le premier est relatif au décalage entre l'activité exercée par un acteur et son projet de vie, le second s'intéresse au décalage entre la nature du projet et le style de l'acteur, le troisième se situe en rapport avec les écarts en terme de savoirfaire entre ce que nécessite la situation et ce que possède l'acteur en situation et le quatrième est relatif au contexte collectif non propre à permettre l'échange et le dialogue vis-à-vis de la situation. En conclusion nous proposerons quelques pistes en matière de gestion des ressources humaines pour tenter de pallier ces obstacles.
\end{abstract}

Mots-clés

Management des situations extrêmes - Perception de l'erreur Apprentissage organisationnel - Organisation apprenante - Services d'Incendies et de Secours

Abstract

This contribution identifies several human factors which represent some limits for an organizational learning in a fire and rescue services. We are interested in the first stage of organizational learning process with the perception of error by an actor in situation (Argyris, Schön, Senge). This research is based on an observation "in situ" (inside a group) of a fire forest operation in French Riviera. We identify four registers which represent limits of an organizational learning. The first register is concerning differences between actor's activity and his own life project. We want to study the motivation and the implication of an actor within his activity. The second one is interested in relation between actor's culture and the kind of project. The third one is concerning the know-how to act in situation. We want study differences between the know-how required to act and the human skills in situation. The fourth register is interested in the community's environment

Revue POLITITQUES ET MANAGEMENT PUBLIC, Volume 26, n², 2008

(C) Institut de Management Public - 2008 
and the collective context. There are some specific conditions which improve organizational learning as social interactions (communication) and common culture. To finish, we present some tracks for a management in specific situations and we try to mitigate these limits for a better organizational learning in risk organizations.

Key Word

Auteurs
Risk management - Perception of error - Organizationnal learning Learning organization - Fire and rescue services

Anaïs Gautier est doctorante en Sciences de Gestion (allocataire de recherche - moniteur 3éme année) à l'Université Aix Marseille II au CRETLOG. Elle prépare actuellement une thèse sur le statut du retour d'expérience dans le pilotage desorganisations en situations extrêmes appliquée au cas des services d'incendies et de secours. Dans sa thèse, elle s'intéresse plus particulièrement aux pratiques opérationnelles $d u$ commandement dans la gestion du risque feu de forêt. Elle est donc amenée à travailler en collaboration étroite et régulière avec la direction du bureau des opérations de l'Etat-major de Zone de Défense Sud (Gardannes, 13) et la direction du groupement des services opérationnels du Service Départemental d'Incendie et de Secours des PyrénéesOrientales (66) ${ }^{1}$. Contact : anaisgautier@hotmail.com

Pascal Lièvre est Maître de Conférences (HDR) en Sciences de Gestion à I'IUP de Management de I'Université d'Auvergne. II est co-responsable du programme de recherche "Management des Processus Organisationnels" au sein du Centre de Recherche Clermontois en Gestion et Management (EA 3849). Ses recherches portent notamment sur la Logistique des Situations Extrêmes, ainsi que sur les méthodologies de retour d'expérience. Les expéditions polaires constituent le terrain principal d'investigation pour des raisons méthodologiques et théoriques, elles sont abordées en tant qu'action collective relevant d'un mode projet sous un angle générique. Il est aussi directeur de recherche au CRET-LOG à Aix en provence. Contact : pascallievre@wanadoo.fr

Géraldine Rix-Lièvre, membre du PAEDI (EA 4281), Eq ExPraCor, est Maître de Conférences en Sciences et Techniques des Activités Physiques et Sportives à l'Université Blaise Pascal (Clermont-Fd). Ses recherches portent notamment sur des méthodologies d'explicitation de l'activité en situation et de retour d'expérience dans différents contextes (arbitrage, situations extrêmes). Elle étudie aussi les risques vécus par des acteurs en situation.

Contact : Geraldine.rix@univ-bpclermont.fr

\footnotetext{
${ }^{1}$ L'étude de cas utilisée dans cet article ne fait aucunement référence au SDIS 66.
} 
au sein de la sécurité civile :

une mise en perspective en termes de gestion des ressources humaines

Introduction
L'objet de cette contribution est d'identifier un certain nombre de facteurs relevant de la gestion des ressources humaines qui font obstacle à l'apprentissage organisationnel dans l'organisation de la sécurité civile en France. Nous focalisons notre attention plus particulièrement sur les registres qui conduisent les acteurs à ne pas percevoir les erreurs en situation.

Au regard de sa complexité, ce secteur constitue un terrain privilégié d'étude des mécanismes d'apprentissage organisationnel, et plus particulièrement de ce qui peut faire obstacle à ce type de dynamique. En effet, il s'agit de gérer des situations d'urgence en coordonnant les actions d'organisations variées dans un contexte de risque et d'incertitude. La capacité des acteurs à faire face en situation est mise à l'épreuve à chaque fois, d'autant plus que le collectif constitué, suite à une alerte imprévisible, est une recomposition permanente d'acteurs et de moyens, et que celle-ci se fait dans l'urgence. De plus, cette recomposition se réalise dans un contexte de transformation profonde de l'organisation des services secours incendie via la départementalisation: le passage d'une institution républicaine inscrite dans un territoire local, reposant sur des valeurs citoyennes, altruistes et de volontariat à une organisation départementale de service public orthodoxe (Padioleau, 2002).

Nous montrerons que la mise en œuvre d'un processus d'apprentissage organisationnel repose sur une étape première qui est la perception par au moins un acteur d'un décalage, dans une situation donnée, qui fera l'objet d'une interprétation en termes d'erreur. Cette perception en situation est fortement dépendante en amont de thématiques qui relèvent du champ de la gestion des ressources humaines. Nous pouvons évoquer ici : le degré d'engagement et des motivations des acteurs dans leurs activités, la compatibilité des cultures professionnelles lors des modalités de coordination, le degré de cohérence entre l'affectation d'un acteur à une mission en référence à son savoir-faire effectif, la nature du climat de travail propre à libérer la créativité des acteurs...

L'organisation de la sécurité civile relève de ce que nous appelons le management des situations extrêmes. Les recherches, autour de cette thématique, se multiplient aussi bien dans la communauté francophone (Amalberti, 1996 ; Journé, 1999 ; Roux-Dufort, 2000 ; Bourrier, 2001; Lagadec, 2002 ; Berry, 2005 ; Kervern, 2005 ) qu'anglophone (Reason, 1983 ; Perrow, 1984 ; Vaughan, 1996 ; Roberts, 1999 ; Weick, 2001 ; Pettigrew, 2002 ) sous des appellations diverses: gestion de crise, cindyniques ou sciences des dangers, gestion de projet à risque, management des risques, management de l'extrême, organisation de la fiabilité... Nous développons dans cet esprit depuis 2000, un programme de recherche de logistique des situations extrêmes, qui s'est centré prioritairement sur le terrain des expéditions polaires ${ }^{2}$ et qui se développe aujourd'hui sur celui de l'organisation de la sécurité civile (Lièvre, 2001,

\footnotetext{
${ }^{2}$ Ce programme prend pour objet la conception et la mise en œuvre d'actions collectives, prenant la forme d'un projet dans un contexte de situations extrêmes; l'action collective est abordée sous un angle délibérément organisationnel, tant dans ses dimensions humaines que techniques. Les expéditions polaires sont abordées comme des études de cas sous un angle tout à fait générique.
} 
Fabbe-Costes, Lièvre, 2002 ; Lièvre, 2003; Lièvre, 2004 ; Lièvre, Rix, 2005 ; Lièvre, 2005 ; Lièvre, Lecoutre 2006 ; Gautier, 2007 ; Rix, Lièvre, 2008).

Précisons maintenant ce que nous entendons par une situation extrême. Une situation extrême est une situation de gestion au sens de Girin (1990) qui présente quelques caractéristiques en matière d'évolution, d'incertitude et de risque. Une situation de gestion se présente selon Girin (1990 p142) «lorsque des participants sont réunis et doivent accomplir dans un temps déterminé, une action collective conduisant à un résultat soumis à un jugement externe ". Nous la qualifions d'extrême lorsqu'elle présente trois caractéristiques : elle est évolutive, incertaine et risquée (Lièvre, 2005). Une situation "extrême " est avant tout une situation évolutive; ainsi elle apparaît pour les acteurs en cause comme présentant une certaine rupture par rapport à un mode de fonctionnement antérieur (Rivolier, 1998). Deuxième notion, le contexte apparaît pour les acteurs comme incertain. Nous sommes ici en présence d'une incertitude radicale où l'imprévisible est possible comme l'exprime Le Moigne (1990). Troisième critère, nous sommes en présence d'une situation à risque c'est-à-dire une situation où la possibilité qu'un évènement non souhaité survienne ne peut pas être écartée et que celui-ci cause des dommages plus ou moins importants à l'organisation.

Nous considérons que l'organisation de la sécurité civile relève de ce que nous avons appelé une situation extrême de gestion: rupture entre une situation avant et après une alerte, émergences d'éléments imprévisibles dans le déroulement des opérations, présence de risques pour les territoires concernées par l'alerte, qui peuvent entraîner la perte de vies humaines et la dégradation de l'environnement, mais aussi pour les opérateurs de la sécurité civile. Une opération de la sécurité civile peut être considérée comme une situation extrême de gestion " contrainte», pour les participants et présentant des caractéristiques de gestion d'urgence. Non que les différents personnels de la sécurité civile n'aient pas choisi librement cette activité, mais ils se trouvent à faire face à des évènements extérieurs à euxmêmes, par exemple, un feu de foret et ceci dans l'urgence. De plus, les activités des services d'incendies et de secours sont fortement encadrées par une réglementation, par une institution où les personnels sont formés et par un management hiérarchique et pyramidal. En effet, une opération de secours implique la mise en œuvre d'une organisation éphémère encadrée par la loi du 3 mai 1996 avec l'attribution d'une compétence exclusive aux Services Départementaux de Secours et d'Incendie (SDIS) dans le domaine de la prévention, de la protection et de la lutte contre les incendies (Cour des Comptes, 2004). C'est pourquoi l'organisation des interventions est définie par des doctrines telles que la Marche Générale des Opérations qui comporte les différentes étapes que sont: la reconnaissance des lieux du sinistre préalable à tout engagement de moyens afin de définir la mise en place du dispositif assurant la sécurité des intervenants et l'efficacité des actions ; la mise en sécurité qui correspond à la protection des enjeux et notamment aux sauvetages des victimes potentielles ou avérées ; l'attaque du feu dont l'objectif est l'extinction au moyen d'une sectorisation du feu (tête ou front de flamme, flanc droit, flanc gauche, queue) pour une répartition des missions tactiques et enfin la surveillance après l'extinction pour éviter le risque de reprise. 
au sein de la sécurité civile :

une mise en perspective en termes de gestion des ressources humaines

Dans une première partie, nous ferons état des résultats qui nous amènent à considérer à la suite des propos d'Argyris (2003) que la perception de l'erreur constitue une étape préalable à tout apprentissage organisationnel ou que la remise en cause de l'expérience acquise est un prérequis à la construction d'une organisation apprenante (Senge, 2001). Ainsi il est possible de considérer la perception d'un écart identifié comme une erreur par au moins un acteur dans une situation comme une étape première à la mise en œuvre d'un processus d'apprentissage organisationnel. A partir d'un travail sur l'histoire exemplaire d'une expédition polaire commanditée par le gouvernement britannique, le plus grand désastre de l'exploration arctique selon Jean Malaurie (1990), nous avons été amenés à distinguer quatre registres en matière de perception d'erreur qui peuvent constituer des obstacles en matière d'apprentissage organisationnel. Nous proposerons dans une deuxième partie, à partir de cette grille, une lecture d'une situation naturelle relative à l'organisation de la sécurité civile lors d'un départ de feux dans le sud de la France. Cette étude de cas est apparue comme exemplaire pour mettre en relief les problèmes d'apprentissages organisationnels de l'organisation de la sécurité civile. En conclusion nous ferons des recommandations en matière de gestion des ressources humaines dans le cadre de l'organisation de la sécurité civile en France pour améliorer les possibilités d'apprentissage organisationnel.

\section{La perception}

de l'erreur comme prérequis à l'apprentissage organisationnel

\section{Retour sur la notion d'apprentissage organisationnel}

Nous ne reviendrons pas dans cet article sur la notion d'apprentissage organisationnel ou d'entreprise apprenante largement développée par des auteurs comme d'un coté Argyris, Schön, et de l'autre Senge. Nous concentrons notre attention sur la question de l'étape préalable à tout apprentissage organisationnel que nous considérons relever de l'ordre de l'individu apprenant avant d'être celui d'un collectif apprenant. En effet, il est possible d'identifier une convergence entre ces différents travaux pour reconnaître qu'il y a en quelque sorte selon Argyris et Schön $(1978,1996)$, une étape préalable à la mise en œuvre d'un processus d'apprentissage organisationnel ou qu'il existe selon Senge (1991, 2001), un pré-requis à la mise en place d'une organisation apprenante. Chez Argyris et Schön, c'est la perception d'une erreur par un acteur qui constitue cette étape préalable. II y a erreur selon Argyris (1993/2003) quand un écart apparaît entre une intention et les conséquences effectives d'une action. Le constat de cet écart est la première étape de l'apprentissage. De fait sans conscience de cet écart, il n'y a pas d'apprentissage. Chez Senge (2001), c'est la remise en cause de l'expérience des acteurs en situation qui pose la question de la perception d'un décalage entre le savoir faire de l'acteur et ce que lui demande la situation. Sans la perception de ce décalage, il n'y a pas de possibilité d'aller vers une organisation apprenante. Nous proposons de revenir sur cette question de la perception d'un décalage dans une situation par un acteur donné. Cette étape doit être considérée comme une condition nécessaire mais pas suffisante de l'apprentissage organisationnel. II faut considérer l'individu apprenant comme la source véritable de 
l'apprentissage organisationnel et essayer d'en dégager toutes les conséquences pour notre questionnement. Nous nous situons ici dans une perspective où, comme l'indiquent Argyris et Schön (1996), l'apprentissage organisationnel repose sur des individus « apprenants » qui interagissent dans un contexte organisationnel spécifique. Mais ces auteurs n'en dégagent pas toutes les conséquences. En remettant l'individu apprenant au centre de l'organisation apprenante, nous devons revenir aux fondements même de l'apprentissage des acteurs en situation. Pourquoi un acteur apprend-il? Dans quel contexte les acteurs sont-ils amenés à apprendre? Le travail que nous avons entrepris dans le cadre des expéditions polaires (Lievre, Récopé, Rix, 2003; Recopé, Rix, Fache, Lievre, 2006), nous a amené à envisager toute situation d'apprentissage, pour un acteur donné, comme s'inscrivant plus globalement dans un phénomène adaptatif au sens de Piaget ou de Vergnaud, dans une perspective bio-logique (Morin, 1980, p454), ayant une dynamique temporelle c'est-à-dire s'inscrivant dans une histoire de vie (Récopé, 2001). Comme le précise Michel Recopé (2001, p11), l'adaptation dépend toujours d'une expérience strictement personnelle dans un contexte particulier : tout individu construit dans l'expérience vécue, le monde auquel il s'adapte. Aussi l'apprentissage survient uniquement lorsqu'une expérience vécue s'accompagne d'un écho mémorable, d'une signification sensible, qui s'inscrit dans une histoire de vie, en référence en une tendance. Une histoire de vie qui se construit, au fil du temps, en termes de stratégies identitaires explicites et implicites, d'expériences corporelles significatives, donc d'acquisitions de connaissances et de savoir-faire : c'est un « monde » de sensibilité et de valeur qui s'élabore. Cette histoire de vie, est orientée, par une suite d'essais et d'erreurs, vers le plaisir, le bien-être " singulier », " en propre» de l'acteur. Ces travaux s'inscrivent dans la lignée de la théorie " relationnelle cognitive motivationnelle » des émotions de Lazarus (2001). Cet auteur propose le terme d'appraisal -qu'on peut traduire par estimation, appréciation, évaluation des objets, événements- en le préférant à celui de "perception ». Ce dernier lui paraît ambigu, dans la mesure où il n'indique pas explicitement une évaluation de la signification personnelle de l'environnement au regard du bien-être. En effet, l'homme apprécie constamment, de manière largement inconsciente, les circonstances internes et externes auxquelles il est sensible, en référence à ses valeurs personnelles, à l'impact existentiel des buts poursuivis, et à ses intentions en situation, et (ré)agit conformément à cette appréciation. Les valeurs personnelles posent le bien-être/le mal-être et déterminent le contenu qualitatif d'une expérience émotionnelle ainsi que son intensité. Lazarus (2001) défend ainsi la rationalité de l'émotion : pour la comprendre, il faut selon lui admettre le poids des intérêts personnels et s'intéresser aux inclinations (manières récurrentes qu'a une personne de sentir et d'agir, structurée par ses valeurs) plus qu'à des états émotionnels évanescents. L'appraisal n'est pas réductible à des formes conscientes et délibératives : c'est une appréciation intuitive portant sur les aspects perçus ici et maintenant, en situation. La perspective est clairement relationnelle : c'est l'appraisal opéré à l'égard des évènements qui guide les conduites, et non les évènements eux-mêmes ${ }^{3}$. Pour ancrer ces réflexions sur des situations

${ }^{3}$ Ces travaux renouent avec les perspectives ouvertes par un philosophe comme Georges Canguilhem (2003) sur les relations entre l'homme et son milieu de vie autour de la question des "normes vitales». 
au sein de la sécurité civile :

une mise en perspective en termes de gestion des ressources humaines

concrètes et répondre ainsi à des critiques déplorant un déficit de travaux empiriques en matière d'apprentissage organisationnel (Huber, 1991), nous avons travaillé sur le plus grand désastre de l'exploration arctique, l'expédition de Sir John Franklin pour la conquête du passage du NW commanditée par la Royale Navy qui a cumulé un nombre d'erreurs " incroyable " dont nous proposons de retrouver la rationalité. Nous serons ainsi amenés à distinguer quatre registres en matière de perception de décalage, en matière d'erreur qui doivent être considérés comme des obstacles à l'amorce d'un processus d'apprentissage organisationnel.

\section{Les leçons en matière d'apprentissage organisationnel de l'expédition de John Franklin}

Un travail sur une situation extrême de gestion particulière, empruntée à l'histoire de l'exploration polaire à savoir le cas du désastre de l'expédition de Sir John Franklin à la conquête du passage du NW en 1850, nous a conduits à définir différents registres pouvant faire obstacles à l'apprentissage organisationnel en remettant au centre de la réflexion l'individu apprenant (Lièvre, Rix, 2005) comme nous venons de le développer précédemment. Cette expédition qui devait couronner le savoir faire britannique en la matière, va aboutir au plus grand désastre de l'histoire de l'exploration arctique : la disparition de deux bateaux de fort tonnage et de leurs équipages soit 120 hommes. Bien que l'énigme ne soit pas complètement élucidée, il est possible sur des faits avérés de dégager des enseignements en matière d'apprentissage organisationnel et de questionner le cadre théorique proposé par les sciences de gestion pour aborder ce type de phénomène. Partant de la thèse que la perception de l'erreur par un acteur dans un collectif constitue le pré-requis à un processus d'apprentissage organisationnel, nous nous sommes interrogés sur la nature des "erreurs " qui ont été commises lors de la phase de préparation et de mise en œuvre de l'expédition, sur ce qui peut apparaître comme une erreur pour un acteur ou un collectif dans une situation donnée... Pour rendre intelligible ce mécanisme, nous avons identifié quatre registres : le premier est relatif à la cohérence entre l'activité exercée par un acteur et son projet de vie, le second est relatif au décalage entre la nature du projet et le style de l'acteur, le troisième est relatif au décalage en terme de savoir-faire entre ce que nécessite la situation et ce que possède l'acteur en situation et le quatrième est relatif au contexte collectif propre ou non à permettre l'échange et le dialogue vis-à-vis de la situation.

\section{L'expédition Franklin selon les quatre « registres » de l'erreur}

a) Le premier registre est relatif au degré de cohérence entre l'activité exercée par un acteur et son projet de vie

Etre choisi en 1850 par la Royale Navy pour diriger une expédition maritime à la conquête du passage du NW est complètement en phase avec le projet de vie de Franklin. C'est un projet où de fait il peut s'engager totalement. On

Nous pourrions aussi relier cette approche en sociologie à la notion d'« habitus » chez Pierre Bourdieu ou en psychologie du travail à celle de « genre » et de « style » chez Yves Clot (2008). 
peut dire que c'est la proposition qu'il a attendue toute sa vie : il a soixante ans. Son modèle de référence est le grand explorateur James Cook. Dés son plus jeune âge, il veut explorer le monde comme un marin à la manière de James Cook. Lorsqu'on lui proposera au début du XIX ème de participer à la conquête du passage du NW en commandant deux expéditions terrestres : il va accepter la mort dans l'âme car ce type de projet n'est pas en cohérence avec son projet de vie. II est clair que courir les bois à pied avec son équipage, apprendre les techniques de survies des indiens, n'a aucun sens pour lui en tant que capitaine de vaisseau de sa majesté. Ces deux expéditions sont des désastres sauvés à chaque fois par le lieutenant Back. De ces deux aventures, Franklin n'aura rien appris parce qu'elles sont à l'écart de son monde. Mais il deviendra un héros populaire de l'exploration polaire : celui qui a survécu en mangeant ses lacets de chaussures. Quant à l'expédition de 1850, il peut percevoir des décalages entre ce qui se passe et ce qui devrait se passer parce qu'il est fortement engagé dans cette aventure.

b) Le second registre est relatif au décalage entre la nature du projet et le style de l'acteur

Comme nous l'avons vu précédemment Franklin, en tant que marin des eaux libres, veut suivre les pas de l'explorateur Cook. Le seul problème pour Franklin est que l'agenda de l'exploration du monde pour l'Angleterre à cette période est l'arctique. Or nous sommes en pleine période du petit âge glaciaire. Le passage du NW est bloqué par les glaces. La présence de la banquise est très basse en latitude et couvre la mer sur une période de plusieurs mois. Ainsi l'exploration polaire suppose une compétence de marin mais aussi de montagnard (les nombreux succès des expéditions polaires norvégiennes attestent cet aspect essentiel), car les bateaux sont pris par les glaces et la solution reste de revenir alors par les terres avec des traîneaux. C'est ce qu'ont fait les capitaines des expéditions maritimes anglaises précédentes celles de Ross, de Parry, ils ont été obligés d'abandonner leurs navires et de revenir par la terre. Aborder la conquête du passage du NW en tant marin des eaux libres n'est pas le bon style pour réaliser cette expédition à cette période. Ce serait un bon style pour réaliser cette expédition aujourd'hui. II ne peut percevoir les décalages entre ce qui se passe et ce qui devrait se passer car il n'a pas le bon style pour réaliser cette expédition. II ne peut penser cette expédition que comme un marin des eaux libres.

d) Le troisième registre est relatif au décalage en termes de savoir-faire entre ce que nécessite la situation et ce que possède l'acteur en situation

Franklin ne perçoit pas les erreurs car malgré son âge et son expérience antérieure en tant que marin et chef d'expédition, on peut le considérer comme un novice pour cette expédition de 1850. II n'a aucune expérience antérieure en tant que chef d'une expédition maritime ayant à naviguer audelà de 70 degré de latitude Nord. Sa seule expérience similaire est celle de jeune second lors d'une expédition écourtée au large du Spitzberg où il entre en conflit avec le capitaine expérimenté du navire sur la stratégie à développer vis-à-vis des icebergs dérivants. Franklin, novice en la matière, ne voit pas le danger d'engager les bateaux face à des icebergs dérivants. C'est d'ailleurs après cette altercation avec son capitaine que la Royale 
au sein de la sécurité civile:

une mise en perspective en termes de gestion des ressources humaines

Navy va l'écarter des expéditions polaires maritimes pendant une vingtaine d'années. Enfin, Franklin part avec un bateau qu'il ne connaît pas et qui doit lui permettre selon lui de slalomer entre les glaces parce que le bateau permet non seulement une navigation à la voile mais surtout pour la première fois à la vapeur.

e) Le quatrième registre est relatif au contexte collectif propre ou non à permettre l'échange et le dialogue vis-à-vis de la situation

Franklin ne peut pas écouter les avis des membres de l'équipage car ils n'ont aucune expérience de navigation en arctique. Le seul qui a une expérience de l'exploration polaire est son second, mais il est trop jeune et trop respectueux de la hiérarchie et de l'expérience de Franklin pour pouvoir émettre un avis. Par ailleurs, Franklin en tant que chef autoritaire, vieillissant, seul maître à bord devant Dieu, n'est pas enclin à aller interpeller son équipage sur les problèmes rencontrés.

Finalement, le seul registre favorable à la perception d'une erreur chez Franklin lors de l'expédition de 1850 est celui qui relève de l'intensité de son engagement, tous les autres registres conduisent au blocage de la situation. La force de son engagement dans cette expédition est orientée vers la seule manière pour lui d'organiser cette tentative celle d'un marin des eaux libres ce qui ne peut le conduire qu'à un échec au vu du contexte climatique de l'époque s'il ne revoit pas sa manière de voir les choses. Mais il faut se rappeler que Franklin a 60 ans lorsqu'il entreprend cette expédition de 1850 et qu'il a un besoin formidable de reconnaissance en tant que commandant d'une expédition maritime de la Royale Navy depuis qu'il a été écarté de l'exploration polaire. Franklin ne peut changer de style ce qui va le conduire au désastre de son expédition.

\section{Une mise en perspective théorique de cette histoire exemplaire}

Essayons maintenant de caractériser ces différents registres sur le plan théorique dans l'objectif de relever ce qui pour un acteur peut faire obstacle à la perception d'un décalage dans une situation. Sur le plan théorique, le niveau 1 et 2 renvoie à la théorie de l'appraisal de Lazarus (2001). Dans le niveau 1, l'acteur ne perçoit pas de décalage dans la situation parce qu'il n'est pas « affecté » par elle. Fondamentalement, il ne s'intéresse pas à ce qu'il entreprend parce que cette activité n'est pas « vitale » pour lui, elle n'est pas essentielle en rapport avec son projet de vie. Dans le niveau 2, l'acteur ne perçoit pas décalage dans la situation parce que son style, sa manière d'aborder le problème est trop à l'écart de ce que nécessiterait la situation. La configuration du projet telle qu'elle a été définie ne correspond pas à son style. Il faudrait que l'acteur remette en cause fondamentalement son expérience, sa manière de "faire les choses » pour percevoir les erreurs. Le registre 2 correspond à l'existence de norme vitale (son habitus dirait Bourdieu) que chacun développe au cours de sa vie et qui contribue à définir le style d'un acteur en rapport avec une activité. Le niveau 3 renvoie aux premiers travaux de Lazarus et Folkman (1984) sur le stress et les capacités des acteurs à faire face à une situation donnée qui dépasse leurs ressources. Le novice ne peut pas percevoir ce qui ne va pas dans la situation car le décalage est trop grand entre son savoir-faire et celui que 
nécessite la situation ce qui va se traduire par une émotion négative violente, le stress. Le jeune étudiant de médecine est incapable de lire la radiographie des poumons de ce patient et encore moins capable de lui proposer une prescription ce qui ne pose aucun problème au professeur qui l'encadre. Enfin le niveau 4 renvoie aux principaux travaux en matière d'apprentissage organisationnel (Argyris et Schön, Senge...). Ce sont tous les obstacles liés au contexte organisationnel qui vont faire que cette perception d'un décalage par un acteur est mort-né pour des raisons différentes: stratégiques, défensives. Le processus d'apprentissage organisationnel ne peut toujours pas démarrer.

Nous proposons d'utiliser cette grille pour étudier les obstacles en matière d'apprentissage organisationnel dans l'organisation de la sécurité civile en France à partir d'une situation naturelle, un départ de feu de forêt, particulièrement révélateur de dysfonctionnement.

Le cas du feu de forêt dans un département du Sud de la France
L'étude de cas $^{4}$ concerne un feu de forêt qui a eu lieu dans le sud de la France. Le schéma suivant présente les principaux protagonistes, leur appartenance et leurs interactions dans la gestion de l'événement en question.

Cf. Annexe 1 - Schéma : Modélisation des interactions au cours de la gestion opérationnelle

\section{Présentation des organisations et des agents}

L'organisation de la sécurité civile en France se compose de différents échelons du niveau national au niveau départemental en passant par un niveau intermédiaire qui est le niveau zonal. Dans le cas qui nous concerne deux échelons sont en interaction : l'échelon zonal qui renvoie à l'Etat-Major de Zone $(E M Z)^{5}$ et l'échelon départemental à travers le Service

\footnotetext{
${ }^{4}$ Pour cette étude de cas, nous avons travaillé a posteriori en procédant à un travail de retranscription minutieux des écoutes de bandes sons (radiophoniques et téléphoniques) enregistrées. Le travail de retranscription de ces écoutes de manière chronologique permet une reconstitution fine des événements a posteriori laquelle est agrémentée par: a) la connaissance du terrain construite au fil d'une observationparticipante au cours de deux campagnes estivales feux de forêts (mois de juin à septembre) au sein des organisations de secours. Cette immersion permet de mieux appréhender d'une part les cellules de décision qui agissent en aveugle et en fonction des messages qui leurs sont transmis, d'autre part le contact direct du feu sur le terrain. b) l'exploitation des mains-courantes qui sont établies pour chaque événement et se composent de courts messages sur lesquels les agents des cellules de décision indiquent la chronologie des actions d'une opération afin d'en conserver la traçabilité en cas d'enquête judiciaire et d'en diffuser l'information à l'échelon national.
}

${ }^{5} C^{\prime}$ 'est un organisme interministériel dont la compétence est constituée par la défense civile et la défense économique, ainsi que par la sécurité civile pour le domaine opérationnel. Pour assurer ses fonctions opérationnelles (veille opérationnelle et gestion de crise), l'état-major de zone intègre un Centre Opérationnel de Zone (COZ). II s'agit d'une cellule de décision constituée d'une salle opérationnelle armée par des personnels issus des FORmations Mllitaires de la Sécurité Civile (FORMISC), des sapeurspompiers et de cadres issus d'autres administrations (police, douane, armée). Parmi les personnels 
au sein de la sécurité civile :

une mise en perspective en termes de gestion des ressources humaines

Départemental d'Incendie et de Secours (SDIS) ${ }^{6}$. Ces deux échelons et les différentes structures spécifiques ne sont amenés à agir ensemble que pendant la gestion opérationnelle des événements sur le terrain autrement dit de manière très ponctuelle. En effet, lorsque le SDIS dispose des moyens suffisants pour assurer la gestion opérationnelle de l'événement, ses relations avec l'EMZ se limitent à une remontée d'information sur les actions engagées et l'état de la situation pour permettre au Centre Opérationnel de Zone (COZ) d'être informé de l'évolution des faits et d'anticiper sur ses actions au besoin. Dans le cas où le SDIS n'a pas les capacités opérationnelles lui permettant de faire face à l'événement, il fait part de sa situation au COZ, ce dernier ayant pour objectif de déterminer la nature des moyens en renfort nécessaire, d'en trouver les ressources à l'échelle zonale et, au besoin, de procéder à un arbitrage pour définir les priorités tactiques pour l'engagement des moyens nationaux en fonction des besoins opérationnels répartis sur l'ensemble de la zone (notamment en saison "feux de forêt " où plusieurs événements peuvent avoir lieu en même temps dans différents départements). Enfin, le COZ doit assurer la coordination dans la mise à disposition des renforts au SDIS bénéficiaire. Ainsi, nous observons que la gestion des flux d'information pour la conduite des actions se situe au cœur du fonctionnement de l'EMZ.

Les principaux protagonistes du cas qui nous occupe sont respectivement: a) l'Officier De Permanence (ODP) du COZ d'origine militaire au grade de major chargé de prendre l'appel du SDIS pour une demande de renfort en moyens aériens; b) le Chef de Salle (CS), adjoint de l'officier départemental, un sapeur-pompier au grade d'adjudant-chef chargé de prendre contact avec le COZ pour la passation de l'alerte rouge correspondant à une demande expresse de moyens en renforts aériens. L'essentiel de l'action va se jouer entre ces deux acteurs, la hiérarchie de chaque organisation venant s'impliquer au fur et à mesure des incompréhensions, et les communications avec la base aérienne et les pilotes intervenant aussi progressivement.

FORMISC, nous relevons la présence d'officiers de permanence pour la prise de décision relevant des événements de gestion courante. II est ici question de l'Etat-Major de Zone de défense sud. Son domaine de compétence concerne treize départements autour de l'arc méditerranéen (de Nice à Perpignan). La gestion opérationnelle des feux de forêt représente une part importante de son activité et constitue une spécificité unique par rapport aux six autres zones de défense.

${ }^{6}$ La loi du 3 mai 1996 définit ses missions : une action préventive pour l'anticipation et la préparation dans la possible survenue d'un événement quelle qu'en soit la nature et une action curative pour la protection et la lutte contre les incendies (Rapport thématique $n^{\circ} 11$ de la Cour des Comptes, 2004). A cela s'ajoute des missions plus larges avec le secours d'urgence à personne dans le cas d'accidents, de sinistres ou de catastrophes et leur transport sanitaire (évacuation terrestre ou aérienne) vers des sites hospitaliers. Ainsi, le SDIS est donc une structure spécifique, locale et dédiée, au contact de l'action opérationnelle des sapeurs-pompiers. Ce service dispose d'un Centre Opérationnel Départemental d'Incendie et de Secours (CODIS) dont la fonction consiste à mobiliser des moyens locaux et à renseigner le $\mathrm{COZ}$ ainsi que les autorités locales pour tout événement d'une certaine ampleur ou à caractère particulier. 


\section{Histoire de vie et contextualisation de l'action}

Avant toute chose, il nous semble important de raconter l'insertion de l'officier de permanence au sein de l'état-major et la situation dans laquelle il se trouve au moment même de l'opération. Le major est un sous-officier issu de la FORmation Mllitaire de la Sécurité civile (FORMISC) relativement jeune pour son grade et qui bénéficiait d'une forte reconnaissance dans son organisation militaire. Sa venue à l'état-major est marquée par une initiative volontaire et une désignation par ses supérieurs pour une fonction en salle opérationnelle. II est donc mis à disposition par les FORMISC pour cette fonction. Son intégration implique un apprentissage de sa fonction d'officier de permanence en salle opérationnelle, celui-ci se faisant par compagnonnage pendant plusieurs mois avant une prise de fonction officielle en tant qu'officier de permanence adjoint. Cette fonction permet de bénéficier d'un apprentissage continu grâce à la présence de l'officier de permanence dont certains sont assurés d'une grande expérience (20 ans de fonction). Nous savons que cet acteur dispose d'un passé relativement récent dans sa fonction puisque nous l'avons vu intégrer l'organisation un an avant au cours de la campagne estivale. Pour lui, la gestion opérationnelle du feu de forêt apparaît comme un élément nouveau dans la mesure où la sécurité civile (FORMISC) intervient sur tous types d'événements de grande ampleur en France et plus particulièrement à l'étranger (pour des tremblements de terre dévastateurs, tsunamis, inondations, tempêtes tropicales...). Par conséquent, le feu de forêt ne s'inscrit pas comme une particularité à part entière comme cela est le cas pour les sapeurs-pompiers que l'opinion publique et la loi du 3 mai 1996 nomment couramment les "soldats du feu ». II a donc tout à apprendre de ses fonctions au $\mathrm{COZ}$ dans un centre opérationnel interministériel en tant que décideur mais aussi des particularités qui caractérisent la zone de défense sud pour le feu de forêt. Ici il ne bénéficie plus de la même reconnaissance parce que sa position d'apprenant l'amène à se former donc à être novice dans l'organisation. Son grade impose le respect mais il est en situation d'apprentissage.

A cela, il convient d'ajouter un autre élément perturbateur avec la modification de la configuration organisationnelle du COZ lors d'une campagne estivale avec la mise en place d'une cellule opérationnelle pour la gestion des moyens aériens de la sécurité civile. Jusqu'à présent, la gestion des moyens aériens faisait partie intégrante des fonctions de l'officier de permanence mais la recherche d'une meilleure gestion dans les opérations a donné lieu à l'insertion de pilotes et de militaires de l'armée de l'air (contrôleurs aériens) pour leurs compétences aéronautiques. Cette cellule a donné lieu à un changement fonctionnel des agents supprimant notamment le poste de l'officier de permanence adjoint. Cela signifie donc que ces derniers sont devenus des officiers de permanence qui conservent un pouvoir de décision à l'échelon zonal sur les mesures à apporter et leur faisabilité opérationnelle, mais avec une fonction n'incluant plus la gestion opérationnelle des renforts aériens. Lors de l'événement qui nous intéresse, la configuration de l'organisation n'a pas impactée l'événement de manière directe. Néanmoins, elle a amené des officiers de permanence adjoints ou encore en formation à prendre la fonction d'officier de permanence et à se retrouver seul dans la conduite des opérations. 


\section{Récit de l'action}

En début d'après-midi (14h33) par une journée de juillet, le chef de salle du CODIS (CS) appelle l'officier de permanence au COZ (ODP) pour la transmission d'une alerte rouge concernant la demande de moyens de renfort aériens pour un feu de forêt. II demande les canadairs, des avions bombardiers d'eau, pour un feu de broussailles dont l'historique des années 1990 fait craindre un risque important. La suite de l'histoire, dont le schéma 2 rend compte de la chronologie, est présentée en 5 étapes déclinant les difficultés qui sont apparues dans la gestion opérationnelle de cet incendie à différents temps de l'action.

\section{Cf. Annexe 2 - Schéma : Chronologie indicative de l'incendie (synthèse)}

a) De la demande des avions à leur attribution sur l'incendie (14h33-16h23)

L'officier de permanence temporise la demande de moyens de renfort aériens afin d'obtenir plus d'informations sur la situation opérationnelle. II ne souhaite pas engager de moyens trop tôt dans le cas où ceux-ci s'avéreraient inutiles suite à l'action des moyens terrestres déployés par le département. Par ailleurs, dans son message, le chef de salle ne lui transmet pas d'information concernant les enjeux et se trompe dans la transmission des coordonnées cartographiques du feu. II s'agit d'autant d'éléments qui n'engagent pas la confiance de l'officier de permanence sur ce feu, il souhaite des informations fiables et précises.

CS : Ils nous confirment qu'il y a $50 \%$ de broussailles, $50 \%$ de résineux et un vent de 5 nœuds du sud, $2000 \mathrm{~m}^{2}$ brûlés et on te confirme la demande de moyens aériens.

ODP : $2000 \mathrm{~m}^{2}$ mais ce n'est pas un feu virulent ca!

CS : Bah ils m'ont dit que c'est dans un talweg, très difficile d'accès et qu'il va partir en crête. C'est pour cela qu'il vous confirme la demande de moyens aériens.

$O D P$ : Il est positionné où là ce feu?

CS : Il est positionné en bout de quartier et il progresse en direction du lieudit $Z$.

ODP : Ah, attends, c'est en B8.2.

CS : Ca c'est le départ de feu et maintenant, il se dirige juste au-dessus en MD06Z.3.

ODP : Ah, il est orienté Est alors

CS : II m'a dit qu'il est orienté sud vers le nord.

OA (hors TPH) : mais il a sauté la nationale le feu alors?

CS : Mais il n'y a pas de nationale.

ODP : Les coordonnées sont fausses dès que tu me les a donné au départ. CS : Ah non !! mes coordonnées ne sont pas bonnes, je me suis trompé !! ODP : Ah ouais, mais attention les gars!!!

CS : Je me suis trompé.

ODP : Et ouais mais ca change tout là, on regarde tout ici nous sur la carte.

Néanmoins, l'officier de permanence prévient le personnel de la base aérienne de la sécurité civile qu'il a une demande pour des canadairs. Le 
personnel de la base a les moyens de satisfaire sa demande, les moyens sont prêts. Le décollage des avions est prévu dans une heure, le temps aux pilotes de se rendre sur base et de se préparer. L'officier de permanence temporise toujours la demande du département, il attend des informations plus précises du chef de salle. Le personnel de la base aérienne soulève l'idée d'une investigation locale, autrement dit d'une reconnaissance aérienne de la situation avec un hélicoptère pour confirmer la nécessité ou non des avions bombardiers d'eau.

Une heure passe depuis la transmission de l'alerte, l'officier de permanence contacte le chef de salle du département afin de savoir s'il a engagé d'autres moyens aériens sur l'incendie. Le chef de salle lui indique que l'hélicoptère de la sécurité civile est déjà en fonction pour une mission sanitaire et que les hélicoptères bombardiers d'eau loués pour la saison estivale ne sont pas encore activés. II n'a donc pas de vecteurs aériens lui permettant de faire une reconnaissance de la situation rapidement. Néanmoins, le chef de salle lui précise un renforcement du dispositif terrestre avec trois groupes d'intervention supplémentaires. L'officier de permanence comprend que l'affaire est sérieuse, il décide de confirmer l'engagement des avions. Ils seront accordés. Le décollage a lieu dans 15 minutes et il faudra 45 minutes aux avions pour se rendre sur les lieux.

Au moment de son décollage, l'un des pilotes avertit l'officier de permanence d'un départ de feu à proximité de X. II décide de survoler la zone en reconnaissance. L'officier de permanence lui demande un point de situation sur ce qu'il observe avant de lui confirmer sa mission pour l'incendie du département $\mathrm{V}$.

Ensuite, l'officier de permanence (ODP) avertit son supérieur hiérarchique des actions menées et de la situation. Cela fait alors une heure trente que l'événement a commencé, c'est l'un des premiers feux de la saison, un de ceux qui signalent si la saison sera virulente ou non en fonction de la vitesse de propagation du feu et de la difficulté à le gérer. Son supérieur (Chef COZ)) lui indique qu'il aurait dû anticiper davantage dans le décollage des avions afin de limiter tout risque de dégradation ou de situation urgente.

ODP : Il y a un départ de feu pour 10 kilo nord-est de $X$. On a engagé les avions.

Chef COZ : Euh oui, tant qu'à faire!

ODP: Oui mais au départ, j'ai attendu un petit peu parce qu'ils ne m'ont annoncé que $2000 \mathrm{~m}^{2}$. II n'y avait qu'un groupe d'intervention au contact et puis tout ça. Donc là, on les a fait décoller donc ils sont en l'air.

Chef COZ : D'accord.

ODP : Ils ne devraient pas tarder à arriver sur zone d'ici 20 à $30 \mathrm{~min}$. J'ai 2 avions. Voilà et pour l'instant, l'évolution depuis $1 \mathrm{H}$, on en est à deux petits hectares.

Chef COZ : Ils ont décollé au bout de combien de temps les avions?

$O D P$ : Au bout d'une heure.

Chef COZ : Oui, c'est normal.

$O D P$ : Oui, ils sont à $1 \mathrm{H}$ donc c'est normal.

Chef COZ : C'est toujours difficile d'annuler quand on a un feu.

ODP : Oui, au début je leur ai dit de me faire un point de situation avec le commandant des opérations de secours (COS) parce qu'au départ ils ne 
au sein de la sécurité civile:

une mise en perspective en termes de gestion des ressources humaines

m'ont annoncé que $2000 \mathrm{~m}^{2}$ de surface brûlée, pas de vent sur zone, 2 groupes d'intervention au contact et en plus les coordonnées étaient mauvaises. Moi je regardais sur la carte, je ne voyais rien du tout.

Chef COZ : Oui.

ODP : Alors je lui ai dit de me faire un point de situation avec son COS et de me rappeler d'ici à 20 min et puis voilà. Et en plus, ils ne m'annonçaient que de la broussaille donc vous voyez quoi.

Chef COZ: Ouais, ouais. Ce que l'on peut faire au mieux dans ce cas là, c'est de passer l'alerte avion de $1 \mathrm{H}$ à $30 \mathrm{~min}$. Quand on a un premier départ de feu comme ça, premier coup de semonce, même si on a pas dit OK pour les avions, on passe déjà de $1 \mathrm{H}$ à $30 \mathrm{~min}$. Ca nous permet même si on n'a pas mis en alerte les avions de gagner après $30 \mathrm{~min}$ au décollage.

ODP : Oui.

Chef COZ: Voilà, c'est à se souvenir d'autant que ce feu ne doit pas être l'affaire du siècle non plus.

ODP : Non, non, je ne pense pas.

Vingt minutes plus tard, les pilotes arrivent sur l'incendie et indiquent que le feu ne leur semble pas important. Ils vont faire deux largages.

b) La complexité d'une demande pour un moyen héliporté (16h23-16h30)

Le chef de salle reprend contact avec l'officier de permanence. II souhaite mobiliser un moyen héliporté (hélicoptère de la sécurité civile) pour effectuer une reconnaissance sur le feu. L'hélicoptère étant rentré de sa mission sanitaire, il est à présent disponible. L'officier de permanence (ODP) temporise de nouveau indiquant qu'il préfère attendre un compte-rendu des avions parce que la situation ne semble pas importante au vu des informations transmises quelques minutes auparavant par les pilotes. II souhaite limiter le nombre de machines sur la zone. Le chef de salle du CODIS (CS) accepte sa proposition.

CS : En ce qui concerne l'hélicoptère, est-ce qu'on pourrait l'utiliser pour la reconnaissance? II rentre de sa mission et on voulait faire une reconnaissance avec le cadre aéro et le commandant des opérations de secours sur place.

ODP: On va attendre le $1^{\mathrm{er}}$ message du pilote parce que j'ai eu des messages comme quoi ce n'était pas très important.

CS : Oui, d'accord. Oui, oui, j'ai entendu le message.

ODP : Alors avant de faire décoller une machine...

CS : OK, on attend.

ODP : On attend le premier compte-rendu de l'avion comme ça tu l'auras en direct et moi aussi. Lui, il est en l'air donc il peut très bien nous faire le compte-rendu de ce qui se passe.

CS : D'accord, OK, allez on attend.

ODP : Stand-by pour l'instant.

CS : OK, merci, ca marche.

A ce moment là, nous observons une incompréhension entre la demande formulée par le chef de salle qui souhaite un moyen aérien spécifique pour effectuer une reconnaissance et la demande d'un compte-rendu aérien. La 
demande du chef de salle traduit le besoin d'un vecteur aérien qui lui permet de bénéficier d'une expertise pompier pour l'évaluation de l'incendie afin de faciliter la gestion opérationnelle ce qui n'a rien à voir avec un compte-rendu aérien transmis par les pilotes. Cette proposition incomprise donne lieu à l'intervention du directeur adjoint du département au grade de colonel (C), car il ne comprend pas qu'on lui ait refusé l'hélicoptère pour une reconnaissance aérienne. Une discussion s'engage entre eux sur les raisons de cette temporisation.

C: Je viens d'apprendre qu'on a demandé l'hélicoptère pour une reconnaissance aérienne et que nous ne l'avons pas.

ODP : Non, ce n'est pas ça.

$C$ (ton agressif): C'est quoi cette affaire ???

ODP: On a les deux avions en l'air et ils vont nous faire un point de situation.

$C$ : Mais je m'en moque du point qu'il vous donne à vous, moi je veux une reconnaissance aérienne de mon département donc je vous le demande et je l'exige.

ODP (résigné) : Oui, d'accord.

C: Bah voilà mais attendez c'est quoi ca??? On demande une reconnaissance aérienne, que les avions vous fassent un point, j'en ai rien à faire moi. Je veux que mon officier aille voir ce qui se passe sur place. Ca c'est autre chose parce que les avions font un point sur quelque chose mais moi, je veux un point sur le feu. Moi, j'ai un officier de permanence et je veux envoyer cet officier pour qu'il me renseigne et cet officier pour qu'il aille le plus rapidement possible, je veux qu'il ait une reconnaissance aérienne. II y a deux lignes électriques, il y a tout un dispositif à mettre en place parce qu'on n'est pas très bien là-bas et malgré que l'on ne soit pas en risque feu de forêt. C'est pour cela que je veux un appareil.

ODP : Bon et bien on va faire le nécessaire mais ce n'est pas la peine...

$C$ : (il coupe l'ODP en hurlant) : Mais attendez !!! (Ca recommence !! C'est la première fois que sur un feu de forêt où on demande une reco aérienne en hélicoptère, on me la refuse.

ODP : (il s'énerve aussi, position défensive): Non mais l'hélicoptère était en l'air pour une mission aussi

$C$ : Non mais si c'est une mission de secours, il n'y a pas de problème. On privilégie la mission de secours. (...) C'est d'ailleurs pour ça que je ne l'ai pas demandé avant ma reconnaissance à cause de la mission de secours sinon on l'aurait envoyé avant.

ODP : Oui mais euh.....on ne m'a pas précisé comme ça, moi. Le problème est dans la formulation de la demande. Il y a ça aussi que quand je lui dis on a les deux avions en l'air, ils vont nous faire un point de situation. II m'a répondu : bon, OK on attend et puis c'est tout quoi. Bon maintenant, si vous avez un besoin spécifique pour votre reconnaissance, j'en conçois quoi.

$C$ : Je le veux et je vous explique pourquoi !! On est à poil au niveau des moyens parce qu'on a un événement sportif qui nous prend des moyens de partout. On a failli demander des renforts à $Z$, on a 5 groupes d'intervention alors qu'on n'est pas en préventif donc on n'est pas bien sur le plan départemental. On a fait 191 interventions cette nuit dont 146 secours à personnes et moi, j'ai besoin de récupérer du dispositif. Si on peut me dégreffer des groupes d'intervention le plus rapidement possible parce que 
au sein de la sécurité civile:

une mise en perspective en termes de gestion des ressources humaines

le feu ne le nécessite pas et bien c'est autant d'engins que j'engage sur l'événement sportif.

ODP : Bon, moi, mon colonel je ne fais pas de frictions car ça fait un an que je suis ici. Maintenant, je ne veux pas de frictions.

$C$ (calme) : OK, d'accord.

ODP : Maintenant, c'est dans la formulation de moyen, il me demande un moyen pour aller faire la reconnaissance, je lui dis "attend ». On a deux avions sur zone qui vont nous faire un point, un compte-rendu. Maintenant, si vous avez une demande particulière sur le feu....

C (il coupe l'ODP) : Moi, ma demande particulière c'est d'alléger le dispositif le plus vite possible une fois que ça passe. Voilà !!

(...)

ODP (stressé) : Maintenant, si vous avez une demande particulière. II n'y a pas de souci. Je vous donne tout ce que l'on a. Ce n'est pas à moi tous ces moyens. Maintenant c'est peut-être dans la formulation qu'il faut peut-être changer les termes et quand j'ai eu l'adjudant-chef auquel j'ai dit: j'ai les deux avions qui vont nous faire un compte-rendu. Ce n'est pas la peine de faire décoller une autre machine pour obtenir le même type de CR. Maintenant si vous voulez quelque chose de particulier, il n'y a pas de souci. Mais c'est dans la formulation qu'il faut peut-être qu'on change les termes.

C : Ok, pas de problème, mea culpa.

D'un côté, l'officier de permanence justifie la temporisation en insistant sur l'imprécision dans la formulation de la demande et de sa motivation: la formulation de la demande par le chef de salle du CODIS ne lui apparaît pas suffisamment explicite et l'acquiescement de ce dernier à l'idée d'une reconnaissance aérienne par les avions conforte la possibilité d'une temporisation.

De l'autre côté, habitué à l'attribution quasi-permanente et sans justification précise des hélicoptères de la sécurité civile pour une mission de reconnaissance aérienne sur un incendie, le colonel manifeste son mécontentement et son incompréhension. En effet, il lui indique qu'il ne souhaite pas un compte-rendu aérien mais un vecteur aérien pour une expertise pompier du feu. Sa demande concerne un hélicoptère qui, pouvant rester en vol stationnaire au-dessus de l'incendie, permet d'avoir une vision globale du sinistre. II est utilisé par le commandant des opérations de secours (le plus haut gradé des sapeurs-pompiers sur l'incendie) ou l'officier " aéro » qui détermine et « marque » les objectifs que les canadairs doivent atteindre ou encore le positionnement des moyens terrestres et les tactiques nécessaires. Dans cette logique, l'hélicoptère constitue un outil fondamental pour l'action de lutte et cette fonction suppose une expertise " pompier » d'une part et d'autre part, un véhicule permettant un vol stationnaire pour une bonne identification de la situation au sol.

c) Les différences culturelles dans l'emploi des moyens (16h30-16h32)

Au même instant, le supérieur hiérarchique de l'officier de permanence (Chef COZ) prend contact avec l'officier de permanence adjoint (ODPA), 
l'officier de permanence étant en communication. L'officier de permanence adjoint lui expose les problèmes dans la demande de la reconnaissance.

ODPA : Et là, il y a un petit souci avec le colonel $X$ pour l'hélicoptère.

Chef COZ : Oui, bien sûr, il faut lui accorder.

ODPA : Et bah apparemment la demande n'a pas été bien formulée. Le major a dit que les avions étant sur place, ils nous feraient un compterendu.

Chef COZ : Non, non, non. II n'est pas là pour ça l'avion, il doit s'occuper du feu !!

ODPA : Bah oui, mais bon. Ah, ça y est !! Ils sont en train de larguer !!

Chef COZ : Tant mieux, déjà qu'ils ont largué plus tard que prévu. Bon, il ne faut pas hésiter à leur donner les hélicoptères, ils sont là pour ça.

L'officier de permanence adjoint abonde dans le sens du raisonnement de l'officier de permanence indiquant au supérieur hiérarchique que la temporisation de la décision est liée à la présence d'un seul hélicoptère de la sécurité civile sur le département $V$ dont la fonction est sanitaire (réservé à l'usage des secours à personne et transport inter-hospitaliers). II revient sur l'idée que la reconnaissance aérienne pouvait être faite par les avions. Le supérieur lui indique qu'il n'est pas d'accord avec la nature de leur intention pour la temporisation d'un moyen héliporté et que ceux-ci doivent être attribués d'office à partir du moment où ils ne sont pas en mission.

Si l'officier de permanence et l'officier de permanence adjoint considèrent tous deux que les avions sont à même de faire la reconnaissance souhaitée par le département, leur supérieur hiérarchique semble prendre la mesure de la demande qui porte sur un bien matériel et non sur une reconnaissance purement visuelle et aérienne.

d) La satisfaction d'une demande officielle et légitime de la part des officiers du département bénéficiaire (16h32-16h40)

Par la suite, le chef de salle du CODIS reprend contact avec l'officier de permanence pour lui transmettre un point de situation dans le cadre de ses obligations de remontée d'information. A l'issu de son message, l'officier de permanence lui indique qu'il a mal interprété sa réponse pour l'attribution de l'hélicoptère. L'officier lui précise qu'il comptait simplement temporiser la décision le temps que les avions transmettent leurs comptes-rendus. Le chef de salle décide de lui transmettre son supérieur hiérarchique, l'officier CODIS. Une autre discussion s'engage sur la justification de la demande pour un moyen héliporté destiné à une reconnaissance. L'officier CODIS (OC) justifie à nouveau le besoin de l'hélicoptère par la situation opérationnelle du département qui est très complexe à cet instant en raison de l'événement sportif. A l'issu de la discussion, l'officier de permanence accorde l'hélicoptère car il considère que la demande a été clairement justifiée : alléger le dispositif opérationnel sur le feu afin de les engager sur l'événement sportif, et parce qu'elle émane de manière officielle de l'officier CODIS lui-même. 
au sein de la sécurité civile:

une mise en perspective en termes de gestion des ressources humaines

ODP : Là, j'ai eu votre chef de salle à qui j'ai dit que la demande serait en stand-by le temps que les avions fassent leur compte-rendu et en fonction, on verra si on vous donne l'hélicoptère ou non. Ca, je l'ai bien dit au colonel et là, il m'a dit OK mais là, ici, on ne vous refuse rien au COZ. Tout ce que l'on peut donner, on donne mais après pour l'hélicoptère, il m'indique qu'il a besoin d'autre chose et dans ce cas, dans les termes de la demande, il faut spécifier exactement pour quoi on le veut. Parce que si c'est pour faire une reconnaissance....

OC (coupe l'ODP): L'hélicoptère est demandé pour faire une reconnaissance sur ce feu pour lequel on a engagé 5 groupes d'intervention et quand en plus avec le département, on se retrouve devant le fait accompli avec l'événement sportif, on n'arrête pas de fournir des moyens depuis ce matin. Donc aujourd'hui, l'officier Codis que je suis commence à se sentir un peu nu. Plus vite, j'aurais un point de situation sur ce feu, plus vite, je serais rhabillé.

ODP: OK, il n'y a pas de souci mais je pense que le compte-rendu des avions serait suffisant mais si maintenant, c'est pour quelque chose de spécifique, il n'y a pas de souci.

OC : Donc on peut le prendre?

ODP : Oui, si vous le demandez, il n'y a pas de souci.

$O C$ : Donc je vous demande d'utiliser officiellement l'hélicoptère.

ODP : OK, c'est bon.

$O C$ : On peut décoller?

ODP : Oui, oui.

Cette discussion montre que l'officier de permanence persiste dans son raisonnement. D'une part, il reste sur l'intérêt d'une reconnaissance aérienne par les avions avant le déploiement d'autres moyens ; d'autre part il obtient entière satisfaction sur la formulation de la demande: une demande officielle, précise et justifiée.

e) Les représailles de la hiérarchie pour une dynamique d'apprentissage « in situ » (16h40-16h50)

Enfin, l'officier de permanence (ODP) s'inquiète de ce que son supérieur hiérarchique (Chef $\mathrm{COZ}$ ) pense de ses actions et décide de le rappeler afin d'éclaircir la nature des reproches qui lui ont été faits pour la temporisation dans l'activation des avions, la temporisation de l'hélicoptère auquel s'ajoute un troisième reproche sur le retard dans la transmission des informations à son supérieur. Le ton du discours est très autoritaire, les reproches ne sont ni acceptés, ni compris par l'officier de permanence. La temporisation de I'hélicoptère occupe une grande partie de la conversation.

Chef COZ (coupe l'ODP) : Non, on aurait pu faire mieux mais ce n'est pas un souci maintenant.

ODP (agressif) : Mais non, mais attendez !!

Chef COZ : l'hélicoptère vous pouvez l'accorder, c'est pas la peine d'hésiter 100 ans. C'est sûr qu'on peut l'accorder tout de suite.

ODP : Attendez !! Faut tout remettre dans son contexte là. L'hélicoptère vient de se poser parce qu'il était sur un sanitaire. Dans la foulée, ils nous demandent l'emploi de l'hélicoptère pour aller faire une reconnaissance sur 
le feu. Je leur dis d'attendre 5 minutes que les deux avions nous fassent un compte-rendu. Et au vu du compte-rendu, on fait décoller l'hélicoptère ou pas.

Chef COZ : Mais là, je ne suis pas du tout d'accord avec vous !!!

ODP : Pourquoi ?

Chef COZ : Parce que le compte-rendu d'un pilote et le compte-rendu d'un commandant des opérations de secours, ce n'est pas du tout la même chose.

(...)

ODP : C'est pas le COS, c'est son officier Codis.

Chef COZ : Bah oui, mais c'est un pompier et un pompier et un pilote n'ont pas du tout le même travail sur un feu. Donc vous le verrez au fur et à mesure avec le temps mais un avion n'a pas la responsabilité de l'engagement.

$O D P$ : Ce que je veux dire, c'est que ce n'était pas un refus catégorique. II faut comprendre. Je leur ai mis un stand-by 5 minutes.

(...)

Je lui dis d'attendre $5 \mathrm{~min}$. le compte-rendu des avions et de toute façon le colonel m'a dit "mea culpa ". Voilà, je veux dire par là que s'il y a une demande d'hélicoptère spécifique, il faut qu'il la formule. Leur demande, c'est : on a besoin de l'hélicoptère pour faire une reconnaissance sur le feu.

Chef COZ : Ca ne vous semble pas assez spécifique?

ODP : Non !!! Je sais pas, il m'a dit qu'il y avait des lignes, il voulait voir comment ses moyens étaient positionnés et ainsi de suite.

Chef COZ: Bah oui, mais ça, c'est quelque chose que l'avion ne vous donnera jamais.

ODP: Oui, mais ça, ce n'est pas quelque chose qu'ils ont demandé au départ. (...) Si vous me demandez l'hélicoptère pour une reconnaissance alors que 5 min après, je vais avoir les avions. Pour l'instant, on met en stand-by. Après, l'appel des avions, on verra si on vous le donne ou pas mais ce n'était pas un gros refus catégorique.

Chef COZ: Non mais qu'est-ce qu'il vous aurez dit le pilote? II vous aurait donné une surface de feu, la virulence et autre mais ce n'est peut-être pas ce dont l'officier pompier a besoin.

ODP (hurlé) : Qu'il me le précise dans sa demande au départ et puis il n'y aura plus de polémique !!!

Chef COZ: Quand il vous dit que c'est pour faire une reconnaissance, il a tout dit.

ODP : Ah ouais, bah ouais !! II a tout dit, il n'a rien dit.

Cette discussion montre que les différences fonctionnelles dans les reconnaissances faites par les avions et par les hélicoptères d'une part, la différence des besoins entre le centre opérationnel de zone qui peut dans certains cas se contenter d'une reconnaissance par les pilotes et le commandant des opérations de secours au plus près du terrain qui a besoin d'une évaluation précise et expertisée de la situation opérationnelle, ne sont pas perçues par l'officier de permanence.

Finalement, le feu a nécessité une après-midi de travail pour les sapeurspompiers ayant engagé un dispositif terrestre de cinq groupes d'intervention autrement dit 20 véhicules soit 90 hommes en plus des pilotes pour les moyens aériens. Ces derniers ont effectué 36 largages et le feu a pu être 
au sein de la sécurité civile:

une mise en perspective en termes de gestion des ressources humaines

circonscrit par action combinée (air-terrestre) sur une surface de $8 \mathrm{Ha}$. L'hélicoptère de la sécurité civile a été mobilisé trois quarts d'heure pour la gestion des opérations.

\section{Analyse du cas}

Tout au long du déroulement de ce cas, nous notons que l'officier de permanence du COZ, contrairement à d'autres acteurs, ne perçoit pas de décalage entre ce qui se passe et ce qui devrait se passer. Dans la mesure où ce dernier est en charge des décisions concernant l'attribution et la gestion de moyens supplémentaires pour faire face à l'événement, il nous parait intéressant d'éclairer les obstacles à l'apprentissage organisationnel dans ce cas à partir des différents registres que nous avons définis précédemment et d'envisager aussi les conséquences en matière de gestion des ressources humaines.

a) La cohérence entre l'activité exercée par un acteur et son projet de vie

Ici, nous n'avons que peu de matériaux pour documenter ce registre. A priori on ne peut pas dire que les deux acteurs principaux ne sont pas " affectés » par la situation. II faudrait examiner les trajectoires de vie des deux personnages pour saisir la nature du rapport qu'ils entretiennent avec leur travail. Deux éléments laissent cependant penser que l'activité qu'exerce l'officier de permanence, un militaire, est en relative cohérence avec son projet de vie. D'une part, sa nomination à ce poste est issue d'une initiative volontaire : personne ne lui a a priori imposé cette fonction et cette orientation de carrière. D'autre part, la vive émotion qui ressort de l'entretien avec son supérieur hiérarchique à la fin de l'événement, montre qu'il est largement affecté par les reproches qui lui sont faits même s'il ne les comprend, ni ne les accepte. Pour le chef de salle du CODIS, un pompier, on peut s'interroger sur la nature de l'erreur qu'il a commise quant au positionnement du feu. S'agit-il d'une erreur liée à un manque d'engagement dans la situation- ce qui relèverait du registre 1-, au fait qu'il ait une appréhension du territoire plus en termes d'espace vécu qu'en termes de positionnement cartographique-ce qui relèverait du registre 2- ou d'un déficit de savoir faire - ce qui serait du registre 3-? En tout cas il a corrigé son erreur. Mais qu'est ce qu'il a retenu de cette histoire ? Nous ne pouvons répondre en l'état. Dans le registre 1 , nous abordons ici la question de l'engagement d'un acteur en termes d'intensité dans une action en fonction de la cohérence entre cette dernière et son projet de vie. C'est la question de la motivation, de la nature du rapport au travail d'un acteur qui conditionne sa possibilité en situation de percevoir un décalage parce qu'il est affecté par la situation. Ce problème relève de la gestion des ressources humaines: la question du recrutement des personnels, de la mobilité interne, de l'affectation du personnel à des missions, de la politique de la formation... Comme nous l'avons noté dans l'introduction, l'organisation de la sécurité civile via la départementalisation provoque des modifications profondes des trajectoires socioprofessionnelles des personnels pompiers en marginalisant le volontariat qui faisait la force de cette institution. Padioleau (2002) a identifié dans ses travaux trois types de motivations qui se combinent, aujourd'hui, chez les sapeurs pompiers: motivation utilitariste, motivation expressive, motivation altruiste. Si c'est la motivation 
utilitariste qui prédomine, on peut s'attendre de la part de ce type d'acteurs à des situations de non apprenance. En effet, on peut considérer cette motivation comme extrinsèque : ce qui intéresse l'acteur n'est pas lié à l'activité elle-même, mais à ce qu'il peut en retirer c'est-à-dire un revenu' Dans cette perspective, alors on peut s'attendre à ce qu'effectivement les acteurs ne perçoivent pas certains décalages parce qu'ils ne sont pas « affectés » par la situation. La question du recrutement est posée avec une certaine acuité. On ne recherche pas exclusivement une "compétence " mais une personne pour qui cette activité prend un sens particulier dans sa vie ce qui est la condition de son apprentissage en situation. Dans le cadre de la formation, il est aussi possible d'aborder ce problème de la motivation et de la question de la construction du projet professionnel en référence avec un projet de vie.

\section{b) L'adéquation entre la nature du projet et le style de l'acteur}

Cet événement, relatif à la gestion des opérations de lutte contre un feu de forêt, est dirigé par le chef de salle du CODIS qui est un pompier. Les échanges et incompréhensions montrent que l'officier de permanence au $\mathrm{COZ}$ ne parvient pas à s'inscrire dans la logique du projet. L'officier de permanence issu d'une culture militaire apporte une importance prépondérante à la fiabilité des informations, à la précision des demandes, à la conformité de la procédure selon ses normes à lui. Les décisions qu'il prend, les réponses qu'il apporte, l'ensemble de son activité, reposent sur ces critères. Ainsi, il vérifie les coordonnées cartographiques données par le chef de salle; il souligne à maintes reprises, dans différentes discussions, l'importance de spécifier et d'expliciter la demande liée à la reconnaissance aérienne; il n'accorde l'hélicoptère que suite à une demande justifiée émanant de l'officier CODIS. Cette culture organisationnelle l'amène aussi à être très respectueux de la hiérarchie tant dans ses modalités d'échange avec son interlocuteur que dans l'évaluation de ce qui est fait.

Cet officier est confronté à une culture différente, celle du milieu pompier, qui lui est relativement étrangère. L'incompréhension concernant la demande d'un moyen héliporté pour une reconnaissance aérienne en est l'exemple le plus flagrant. Alors que pour l'officier CODIS, le directeur adjoint du SDIS et le Chef COZ, la demande est explicite et spécifique : elle concerne un hélicoptère qui, pouvant rester en vol stationnaire au-dessus de l'incendie, permet d'avoir une vision globale du sinistre et de diriger les opérations, pour l'officier de permanence, en disant «faire une reconnaissance ", " II a tout dit, il n'a rien dit ! ". Ainsi, ce dernier ne perçoit pas de décalage entre ce qui lui est demandé et la réponse qui est apportée par l'action de temporiser dans la mesure où il considère qu'une reconnaissance aérienne peut être réalisée par les pilotes des aéronefs dans une perspective d'économie de moyens. II est dans une culture " militaire ». Au poste où il est, il faudrait qu'il puisse saisir une culture « pompier ». II se pose la question des possibilités de passage entre ces deux mondes de valeurs qui sont aussi des manières de faire. On pourrait imaginer que pour ce type de poste l'officier participe pendant une période donnée à des opérations conduites par les pompiers sur le terrain de façon à permettre une acculturation. On peut aussi penser que la confrontation entre les deux interlocuteurs repose sur deux registres différents de

\footnotetext{
${ }^{7}$ Nous sommes dans un certain type de rapport au travail, proche du mode du retrait chez Sainseaulieu.
} 
au sein de la sécurité civile:

une mise en perspective en termes de gestion des ressources humaines

compétence : des compétences techniques issus du domaine militaire pour l'officier du COZ, et de l'autre coté le chef de salle CODIS qui n'a pas cette connaissance cartographique abstraite, mais par contre qui connaît bien le terrain. Cette connaissance indigène du territoire peut se révéler en situation opérationnelle d'une grande utilité. Comment permettre à ces deux types de connaissances, à ces deux types de compétences de s'enrichir mutuellement? Ceci relève tout à fait de la politique de formation interne de la sécurité civile qui dépend du département de la gestion des ressources humaines.

c) Les savoir-faire que nécessite la situation au regard de ceux que possède l'acteur en situation

Si nous avons observé un décalage entre le style de l'acteur et la nature du projet, il faut aussi souligner qu'étant novice, l'officier de permanence n'a pas connaissance d'une part de l'usage dans l'attribution des moyens, d'autre part des conséquences pour les pompiers de la temporisation qu'il opère. En effet, en l'absence de mission sanitaire en cours, l'attribution de l'hélicoptère de la sécurité civile pour le commandement des opérations de lutte en feu de forêt ne souffre pas de temporisation. II est d'usage de l'attribuer d'office sans délai et sans autre justification. Concernant l'impact de la temporisation dans l'attribution d'un hélicoptère de la sécurité civile, il ne perçoit pas qu'il prive ainsi le commandant des opérations de secours d'un moyen nécessaire à l'élaboration des actions de lutte. Enfin, la non compréhension de la demande du moyen héliporté pour une reconnaissance peut aussi être interprétée comme un manque d'expérience de l'officier de permanence dans la mesure où il ne pratique pas les formulations employées. La question de l'identification fine des compétences que doit avoir un acteur dans une situation donnée relève de ce qu'on appelle la gestion prévisionnelle des emplois et des compétences. II s'agit d'identifier les compétences critiques que doivent détenir certains personnels à certains postes de façon à construire des parcours professionnels, une politique de formation et de compagnonnage à même de répondre aux critères attendus de performance organisationnelle.

d) Le contexte collectif propre ou non à permettre l'échange et le dialogue vis-à-vis de la situation

Concernant les possibilités d'échange offertes ou non par le contexte organisationnel, plusieurs obstacles peuvent être soulignés. Le premier tient aux relations très ponctuelles qui lient les différents protagonistes. En effet, l'officier de permanence n'est amené à travailler avec le milieu pompier et en particulier avec le chef de salle du CODIS qu'à l'occasion d'un feu de forêt dans le sud de la France. Pour un tel événement, ce ne sont d'ailleurs pas forcément les mêmes personnes qui seront en poste, ainsi il est plus difficile de constituer une communauté de pratique. Le second obstacle est lié au fait que les interactions entre les agents ont toujours lieu en phase opérationnelle, donc d'urgence. Ils ne sont en contact que lors de la gestion d'un événement critique au cours duquel l'officier de permanence multiplie les communications avec différents interlocuteurs sans pouvoir prendre du recul. Le troisième est relatif au poids de la hiérarchie. L'organisation très hiérarchique de l'Etat Major de Zone induit que tout en étant relativement novice dans la fonction, l'officier de permanence est en charge de la prise 
de décision concernant l'attribution ou non de moyens de renfort. Ainsi ce qui prime tient moins à l'expérience de l'individu qu'à son grade (En opération de secours, la fonction prime sur le grade). Le quatrième renvoie à la fonction même d'officier de permanence. Dans la mesure où le Centre Opérationnel de Zone est confronté à différents interlocuteurs en fonction des événements, il est de fait à l'interface de différentes organisations (pompiers, police, gendarmerie, SAMU...) ayant chacune une culture, un mode de fonctionnement spécifique, qu'il doit s'approprier pour répondre au mieux à ces fonctions. Le caractère " pluridisciplinaire " de ce poste peut aussi apparaître comme un obstacle à l'apprentissage organisationnel. Mais la question posée dans le registre 4 est celle plus spécifique de l'organisation du travail sous l'angle structurel et hiérarchique. II s'agit d'identifier les boucles perverses qui ne permettent pas aux différents acteurs à l'endroit où ils sont de faire remonter les erreurs perçues dans une perspective de re-organisation du service. La question du clivage des différents statuts (militaire, pompiers professionnels, pompiers volontaires) et des positions afférentes dans l'organisation, la question de la hiérarchie et enfin celle de la judiciarisation des événements critiques peuvent entraîner des blocages stratégiques, défensifs des personnels dans une perspective d'apprentissage organisationnel. 
au sein de la sécurité civile:

une mise en perspective en termes de gestion des ressources humaines

Conclusion
La complexité de l'organisation de la sécurité civile en France pose de nombreux obstacles en matière d'apprentissage organisationnel comme le montre le cas que nous avons étudié. Un cas " intéressant » puisqu'il a fait l'objet d'une demande expresse d'investigation de la part des responsables de l'organisation de la sécurité civile. Un cas " intéressant » du point de vue du management des situations extrêmes parce qu'il s'agit d'un incident, d'un « presqu'accident » (Bourrier, 2001) c'est-à-dire d'une situation naturelle où il est possible d'étudier chronologiquement un dysfonctionnement n'ayant pas effectivement entraîné de conséquences graves, mais qui aurait pu en avoir. En étudiant au plus près le déroulement de l'action en situation, nous avons été amenés à appréhender l'étape première de l'apprentissage organisationnel à savoir ce que nous avons appelé la perception par au moins un acteur d'un décalage, dans une situation donnée, qui fera l'objet d'une interprétation en termes d'erreur ; ce décalage peut se traduire comme un écart entre les intentions et les réalisations (Argyris, 2003) ou entre les capacités de l'acteur et cette même situation (Senge, 2001). II s'agit d'une condition nécessaire mais pas suffisante de la mise en œuvre d'un processus d'apprentissage organisationnel. Nous avons montré qu'à partir des travaux que nous avons engagé sur des situations extrêmes de gestion à partir de lourdes investigations empiriques et en mobilisant les travaux de Lazarus (2001) autour de la notion d'appraisal, nous pouvions distinguer quatre registres en matière de perception de l'erreur. Sans perception de l'erreur par un acteur dans une organisation, il ne peut y avoir d'apprentissage organisationnel. Ces registres constituent des thématiques qui peuvent faire l'objet de politique en matière de GRH en amont des situations.

Dans le cas étudié, il apparaît que l'acteur qui est l'origine d'un certain nombre de dysfonctionnements qui n'ont pas prêté à conséquence grave mais qui évidemment aurait pu l'être, n'a pas appris de la situation. L'intérêt de notre approche est de montrer que quelles que soient les modifications du contexte organisationnel le registre 4 si nous n'intégrons pas les registres 1,2 et 3 qui se situent en amont, l'apprentissage organisationnel ne peut véritablement pas démarrer. Si les problèmes rencontrés dans le cas étudié ne relèvent pas du registre 1. Les deux autres registres 2 et 3 sont concernés. En effet, deux questions se posent pour l'ODP. Premièrement, sa culture professionnelle est décalée par rapport aux opérations mises en œuvre par les pompiers. Fondamentalement, il ne peut pas comprendre les attentes de cette opération de pompier car en situation il ferait lui autrement, à sa manière, comme on lui a appris. Nous sommes dans le registre 2 de l'erreur. II est dans une culture " militaire ». Au poste où il est, il faudrait qu'il puisse saisir une culture " pompier ». II se pose la question des possibilités de passage entre ces deux mondes de valeurs qui sont aussi des manières de faire. On pourrait imaginer que pour ce type de poste l'officier participe pendant une période donnée à des opérations conduites par les pompiers sur le terrain, de façon à permettre une acculturation. Deuxièmement, c'est un novice à ce poste. Son savoir-faire accumulé ne lui permet pas de faire face à la situation. En effet, il a du mal à apprécier l'équilibre à maintenir en fonction de la situation entre une logique de réponse rapide par l'envoi des moyens demandés vis-à-vis d'une situation qui peut se dégrader et de l'autre coté une logique de préservation des moyens pour d'autres opérations à venir. L'échange avec son supérieur hiérarchique est net et sans ambiguïté : il n'aurait pas agit comme cela. II lui 
signifie précisément ce qu'il aurait du faire. Mais il ne comprend pas. Nous sommes dans le registre 3 . Pour faire face à ce décalage, il faudrait que ce novice puisse suivre en tant qu'adjoint, un officier de l'EMZ (chef COZ) pendant une période qui lui permettrait d'apprendre à estimer cet équilibre entre ces deux logiques à partir de nombreux cas concrets. Ces problèmes ont fait l'objet de travaux sur d'autres situations à risque comme les opérations chirurgicales avec le dilemme entre deux logiques: celle du chirurgien qui préfère travailler sur un corps inerte et celle de l'anesthésiste qui voudrait être sur que son patient se réveille (De Keyser, Nyssen, 1993).

Ces questions relèvent pleinement du champ de la gestion des ressources humaines et interpellent celle-ci sur plusieurs aspects: les modes de recrutement, l'affectation des personnels à des postes de travail, la politique de formation, l'évolution des carrières et enfin le climat de travail. Le développement d'un processus d'apprentissage organisationnel pose d'une part la question fondamentale du degré d'engagement, des compétences en acte, des cultures professionnelles des personnels en situation opérationnelle et d'autre part du contexte organisationnel propre à permettre les échanges pendant et après les opérations de terrain. Ces réflexions prennent une intensité particulière aux vues des transformations en profondeur que connaît l'organisation de la sécurité civile depuis la départementalisation (Padioleau, 2002) dans un contexte socio-économique spécifique (société du risque, individualisme, rationalisation budgétaire) et de l'intérêt grandissant de ce type de structure à mettre en œuvre une gestion prévisionnelle des emplois et des compétences. Enfin, espérons que le processus de modernisation de la sécurité civile ne détruise pas les valeurs à l'œuvre chez les sapeurs-pompiers, source d'un engagement des personnels et de la constitution de communautés de pratiques (Wenger, 1998), des catégories " premières » dans la construction des organisations apprenantes. 


\section{BIBLIOGRAPHIE}

Amalberti R., (1996), La conduite des systèmes à risque, PUF, Paris. Argyris C., (2003), Savoir pour agir, Paris.

Argyris C., Schön Donald, (1978), Organization learning, a theory of action perspective, Reading MA : Addison-Wesley.

Argyris C. Schön D., (1996), Organization learning II, Reading MA : Addison-Wesley

Berry M., (2005), Managements de l'extrême, Tome 1, Edition Autrement, Paris.

Bourrier M., (2001), Organiser la fiabilité, L'Harmattan, Paris.

Canguilhem G., (2003), La connaissance de la vie, Paris, Vrin.

Clot Y., (2008), Travail et pouvoir d'agir, Edition PUF, Paris.

De Keyser, Nyssen, 1993, Les erreurs humaines en anesthésie, Le travail humain, vol 56, n²-3, p.233-241.

Fabbe-Costes N., Lièvre P., (2002), « La logistique des expéditions

polaires : caractérisation et apports à la logistique classique », Logistique et Management, $\mathrm{Vol} 10, n^{\circ} 2, p .25-39$

Gautier A., (2007), "Vers une méthodologie de recherche qualitative: le statut du retour d'expérience dans le pilotage des organisations en situation extrême " Conférence Internationale ISEOR \& AOM, Acte CDROME, Lyon, 26, 27, 28 mars, 14p.

Huber G.P., (1991), "Organizational learning: the contributing processes and the literatures", Organization Science, vol 2, p.88-115.

Journé B., (1999), "Les organisations complexes à risque : Gérer la sûreté par les ressources. Etudes de situation de conduite de centrales nucléaires. Thèse de doctorat, Ecole Polytechnique, Paris.

Kervern G.Y., (2005), " Histoire des cindyniques, émergence de nouveaux 'patterns': Déconstruction de la destruction ", Colloque de Cerisy: "Intelligence de la complexité : épistémologie et pragmatique» sous la direction d'Edgar Morin et de Jean Louis Le Moigne, Cerisy, Juin.

Lagadec P., (2000), « Ruptures créatrices », Editions d'organisation - Les Echos éditions, Collection Tendance, Paris.

Lambert B., Gaulier A., Massaiu A, (2000), Retour d'expérience sur incendie touchant des coupures de combustible - Méthode de recueil et analyse de données, Documentation du Réseau Coupures de Combustible (RCC) en collaboration avec Espaces Méditerranéens.

Lazarus R.S., (2001), "Relational Meaning and discrete Emotions", in K.S. Scherer, A. Schorr, \& T. Jonhstone (Eds), Appraisal Processes in Emotion : Theory, Methods, Research, pp 37-67, Oxford/New-York, Oxford University Press.

Lazarus R.S., Folkman S., (1984), Stress, appraisal and coping, New-York, Springer Publishing Company.

Lièvre P., (2004), "Vers un savoir d'action en sciences de gestion : le cas des expéditions polaires ", Annale des Mines, Gérer et Comprendre, $n^{\circ} 75$, mars, p.4-18.

Lièvre P., (2005), Vers une logistique des situations extrêmes, de la logistique de projet du point de vue d'une épistémologie de l'activité d'une expédition polaire, HDR, Université Aix Marseille II. Lièvre P., (2007), La logistique, Editions La Découverte, Collection Repères, Paris. 
Lièvre P., dir., (2001), Logistique en milieux extrêmes, Edition Hermès, Paris.

Lièvre P., dir., (2003) La logistique des expéditions polaires à ski, Editions GNGL.

Lièvre P., Rix G., (2006), « Vers un observatoire de l'organisant, une illustration à partir des expéditions polaires », Colloque " Autour du langage et des organisations " en hommage à Jacques Girin, PREG-CRG, Ecole Polytechnique, CNRS, Carré des Sciences, Paris, 12 novembre 2006, 15p. Lièvre P., Lecoutre M., (2006), « Le processus de mobilisation des réseaux sociaux dans une démarche de projet : la notion de lien faible potentiellement coopératif. Le cas des expéditions polaires », Revue Sciences de Gestion, ${ }^{\circ} 52$, décembre, p 83-106

Lièvre P., Récopé M., Rix G., (2003), « Finalités des expéditeurs polaires et principes d'organisation », In P. Lièvre, La logistique des expéditions polaires à ski (pp.85-101). Paris : GNGL Production.

Lièvre P., Rix G., (2008), « Vers un observatoire de l'organisant : le cas des expéditions polaires, AIMS, Atelier Analyse Qualitative, IAE de Lille, 22 mai, 1-17p.

Lièvre P., Rix G., (2005), "Le management des expéditions polaires ", Revue Française de Comptabilité, 383, Décembre, p.46-52.

Lièvre P., Rix G., (2005), « Organisation apprenante : le cas exemplaire des expéditions polaires ", Colloque de Cerisy "Intelligence de la complexité : épistémologie et pragmatique ", sous la dir., Jean Louis Le Moigne, Edgar Morin, 23-30 juin 2005, site MCX.

Malaurie J., (1990), Ultima Thulé, Edition Bordas, 443p.

Padioleau J.G., (2002), La fin des Sapeurs-Pompiers républicains ?, Edition L'Harmattan.

Perrow C., (1994), "Normal accidents, living with Hight-Risk Technologies, NJ, Basic Book, New-York.

Pettigrew A.M., (1990), "Longitudinal field research on change: theory and practice", Organization Science, 1, 267-292.

Cour des comptes (2004), Rapport thématique 11 «Les services départementaux d'incendie et de secours (SDIS) ", http://www.ccomptes.fr/cc/Sommaire-6.html (rapport SDIS) p.389-438.

Reason J. (1987) "The age of organizational accident", Nuclear Engineering International, 18-19.

Recopé M., (2007), "Conceptualisation et normativité vitale " in Activité humaine et conceptualisation, questions à Gérard Vergnaud, coord. M. Merri, Presses Universitaires du Mirail, Toulouse.

Recopé M., (coord.), (2001), L'apprentissage, Edition Revue EPS.

Recopé M., (2007), "Conceptualisation et normativité vitale », in Activité Humaine et Conceptualisation, questions à Gérard Vergnaud, (Coor.) M. Merri, Presses Universitaires du Mirail, Toulouse.

Recopé M., Rix G., Fache H., Lievre P., (2006), "Sensibilité et mobilisation: perspective d'investigation du sens à l'œuvre en situation pratique », eJRIEPS, 9 janvier 2006, pp.51-66.

Rivolier J., (1998), "Stress et situations extrêmes" Numéro spécial Bulletin de psychologie, tome 51 (6), novembre-décembre.

Roberts K.H., (1990), Managing high reliability organization, Californian Management Review, 32/4, 101-113.

Roux-Dufort C., (2003), "Gestion de crise, dépasser les mythes pour agir » Management et conjecture sociale, $\mathrm{n}^{\circ} 617$. 

au sein de la sécurité civile:

une mise en perspective en termes de gestion des ressources humaines

Senge P., (1991), La cinquième discipline: l'art et la manière des entreprises qui apprennent, Editions Générales First, Paris.

Senge P., (2001), La cinquième discipline: le guide de terrain, Editions Générales First, Paris.

Vaughan D., (1996), The Challenger Launch Decision: Risky Technology, Culture and Deviance at NASA, University Of Chicago Press.

Wenger E., (1998), Communities of practice; learning, meaning and identity, Cambridge University Press, Cambridge. 
Annexe 1 - Schéma : Modélisation des interactions au cours de la gestion opérationnelle

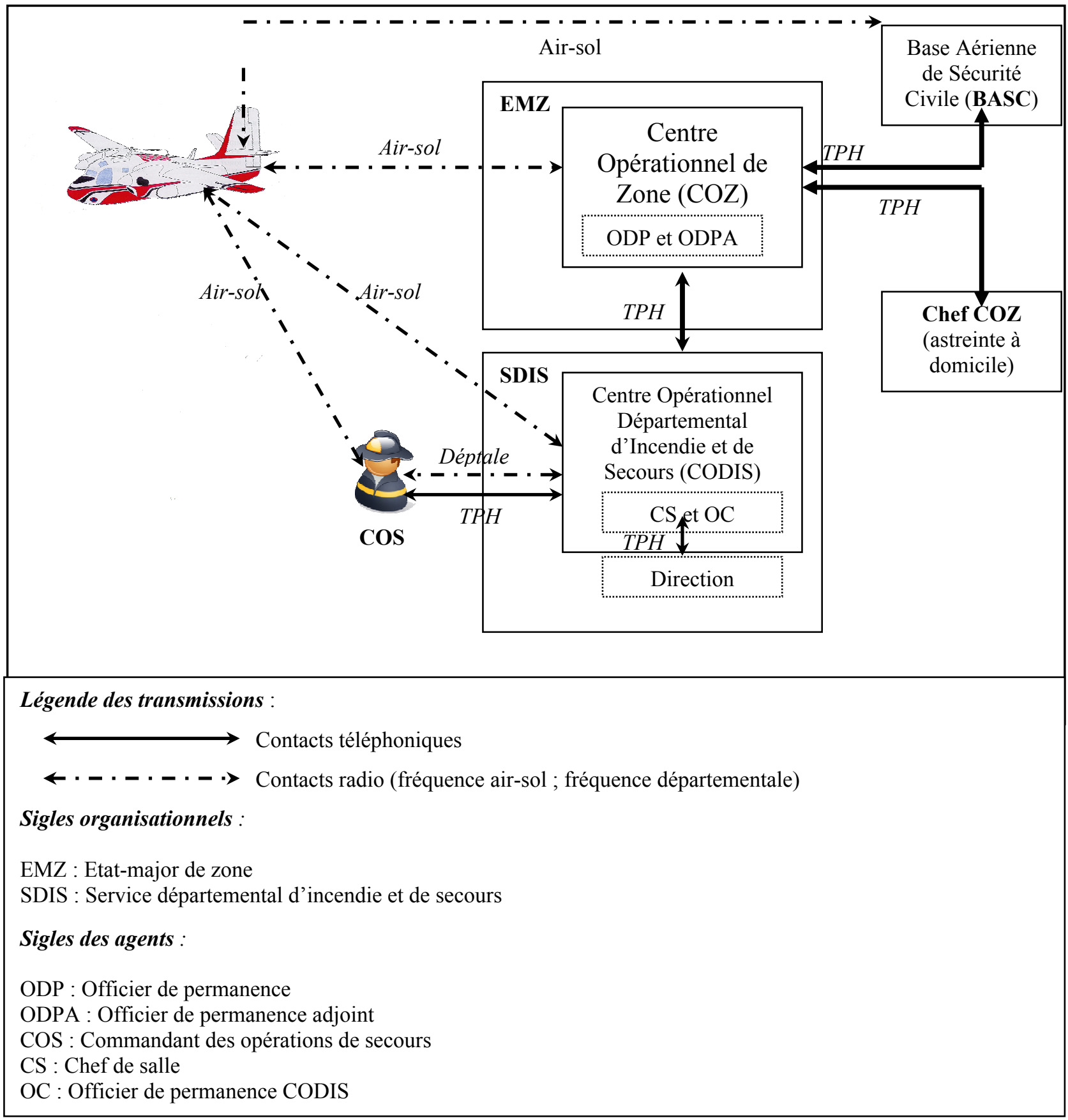




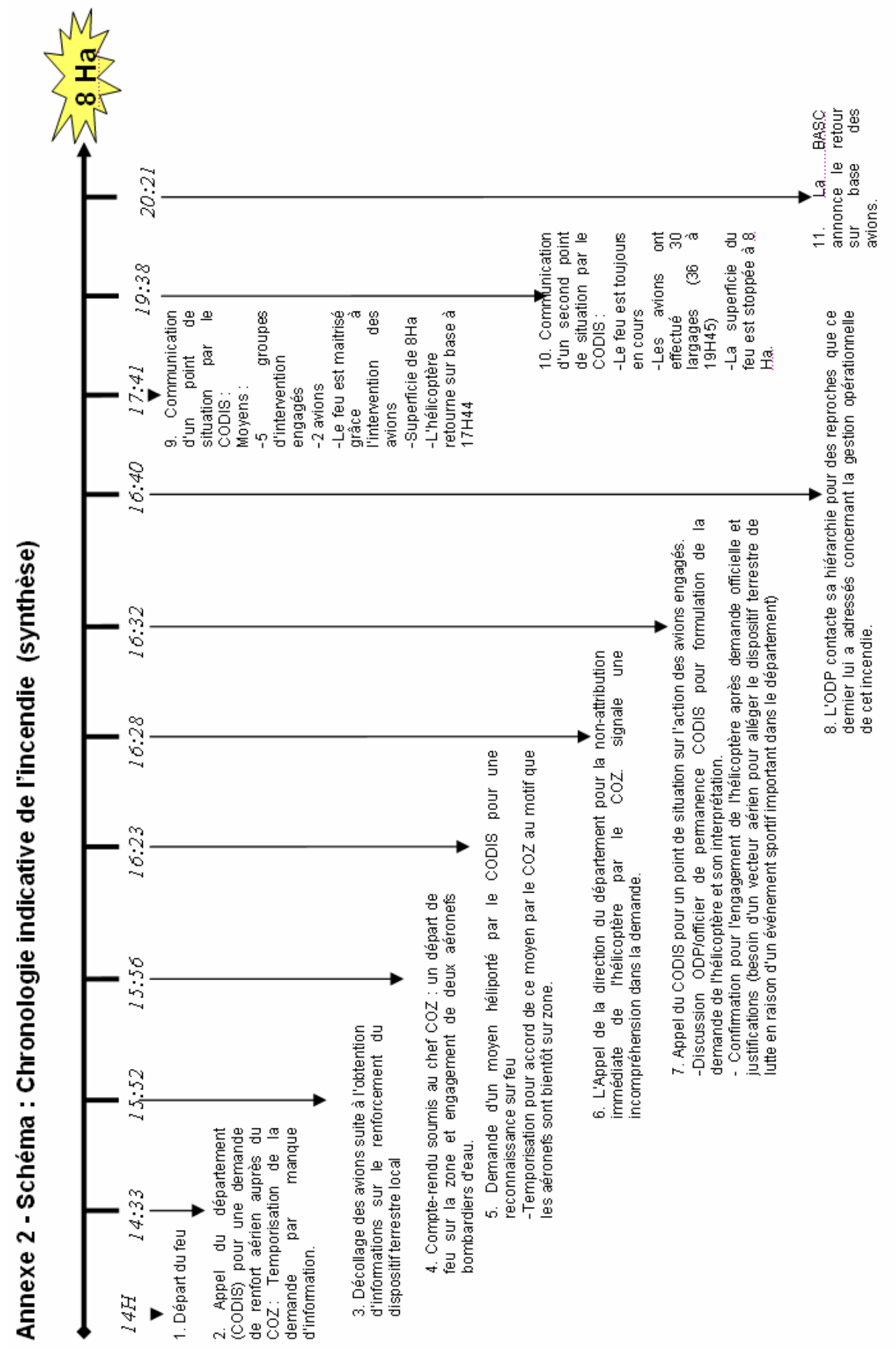

